\title{
Topology Optimization of a Constrained Layer Damping Plate Coupled with an Acoustical Cavity
}

\author{
Zheng Ling, Zhang Dongdong, LiuChengfeng and Li Yinong \\ State Key Laboratory of Mechanical Transmission, Chongqing University, Chongqing, 400044, China \\ Xiang Shuhong, Li Ye and Fang Guiqian \\ Beijing Institute of Spacecraft Environment Engineering, Beijing, 100094, China
}

(Received 26 June 2014; accepted 10 June 2016)

An acoustical topology optimization of a constrained layer damping (CLD) plate coupled with a rigid acoustical cavity is presented to minimize the sound radiation power. A mathematical model is developed to simulate the sound radiation based on the theories of the finite element and boundary element methods together. The model is integrated with the acoustical topology optimization approach, which utilizes the genetic algorithm with an elitist strategy. The obtained results demonstrate the effectiveness of the proposed approach in attenuating the sound radiation power and the sound pressure inside the acoustical cavity simultaneously by proper layout of the CLD materials. Furthermore, experimental verification is carried out by manufacturing topology optimized CLD/plate and monitoring the sound pressure in the acoustical cavity. The experimental results are a good match with the predictions of the mathematical model. The study shows that the proposed acoustical topology optimization approach can be an effective tool in the design of a wide variety of critical structures, which is lightweight and operates quietly, such as the panels in the vehicle body and aircraft cabin.

\section{NOMENCLATURE}

$a, b$
$\mathbf{b}$
$b_{j m n}$
$\mathbf{B}$
$b_{j}$
$C(\alpha)$
$E_{j}^{(e)}, E_{\beta v}^{(e)}$
$f$
$\mathbf{F}$
$G(\alpha, \xi)$
$h_{p}, h_{v}, h_{c}$

$\mathbf{h}$

$\mathbf{H}$

$\mathbf{K}^{(e)}, \mathbf{K}$

$\mathbf{M}^{(e)}, \mathbf{M}$

$\mathbf{N}$

$N_{i}$

$p(\alpha), p_{Q}$

$\gamma_{j x y}$

$\boldsymbol{\delta}^{(e)}$

$\varepsilon_{j x}, \varepsilon_{j y}$

$\theta_{x}, \theta_{y}$ $p(j)$ matrix
Half of the element length

The coefficient matrices to calculate sound pressure at point $\alpha$

Element in the coefficient matrices $\mathbf{b}$

The coefficient matrices to calculate the nodal sound pressure on the boundary surface

Element in the coefficient matrices $\mathbf{B}$

Constants in Helmholtz acoustical boundary integral equation

The potential energy for the element

The fitness function

Externally applied mechanical force

Green's function

The thickness of base layer, damping layer and constrained layer

The coefficient matrices to calculate sound pressure at point $\alpha$

The coefficient matrices to calculate the nodal sound pressure on the boundary surface

Element stiffness matrix and global stiffness
Shape function

Sound pressure at point $\alpha, Q$

The shear strain for each layer

The nodal displacement vector

The strain at the $x$-direction and $y$-direction

Rotations about the $x$-axis and the $y$-axis

Sound pressure at node $j$
Shape function matrix $\mathbf{p}_{m}$

$\mathbf{P}$

The nodal sound pressure vector of element $m$

Nodal sound pressure vector on the boundary surface

$T_{j}^{e} \quad$ The potential energy for the element

$u_{p}, u_{c}, u_{v} \quad$ The displacement at the $x$-direction for base layer, damping layer and constrained layer

$v_{p}, v_{c}, v_{v} \quad$ The displacement at the $y$-direction for base layer, damping layer and constrained layer

$v_{Q} \quad$ The vibration velocity at any point $Q$

$\mathbf{v}_{m}^{*} \quad$ The complex conjugate of the nodal normal vibration velocity vector of element $m$ The nodal normal vibration velocity vector The transverse displacement of the node The sound radiation power

Design variables

The design variable set

The displacement vector

$\alpha \quad$ The field point

$\beta_{x}, \beta_{y} \quad$ The shear deformation at the $x$-direction and $y$-direction of the damping layer

$\xi \quad$ The point on the acoustical field boundary

$\sigma_{j x}, \sigma_{j y} \quad$ The stress at the $x$-direction and $y$-direction

$\tau_{j x y} \quad$ The shear stress for each layer

\section{INTRODUCTION}

CLD treatment has been regarded as an effective way to suppress structural vibration and acoustical radiation since it was proposed by Kerwin. ${ }^{1}$ It has found its ways in aeronautical, vehicle, civil, and mechanical engineering applications. Meanwhile, the optimizations for the layout of CLD materials have been widely reported in recent years because it has been recog- 
nized that using such an approach can significantly improve the static and dynamic characteristics of the structures with CLD treatments.

Literature on the optimization for the layout of CLD materials are quite extensive and the research activities in this field have focused on a variety of applications. Zheng et al. studied the optimal location and length of rectangular CLD sheets using the genetic algorithm based on the penalty function method and the optimization aimed to minimize the volume displacement of the cylindrical shell. ${ }^{2}$ Magnus Alvelid studied the optimal number and location of CLD sheets on flat structure surface and used an improved gradient method; the objective was to minimize the vibration velocity on the structural surface. ${ }^{3}$ Zheng et al. studied topology optimization for the layout of CLD in plates and shells to minimize structural modal damping ratios by using the ESO method, ${ }^{4}$ optimality criteria (OC), and the method of moving asymptotes (MMA). ${ }^{6}$ It was found that the ESO and OC methods resulted in a fast optimization for the layout of CLD on the plate by using a small compute effort. On the other hand, the MMA method obtained more accurate and optimal results than the previous two methods. However, a higher computational effort must be paid. Ansari et al. adopted a novel level set method to search the best shapes and locations of CLD patches on a cantilever plate. ${ }^{7}$ The main goal was to maximize the modal loss factor of the system and it was found that the proposed method would increase the modal loss factor of the system through shape change from a square to a circle. Kim et al. compared modal loss factors obtained by topology optimization to the conventional strain energy distribution (SED method) and the mode shape (MSO approach) ${ }^{8}$ It was found that topology optimization based on the rational approximation for material properties (RAMP) model and optimality criteria (OC) method could provide about up to a 61.14 percent higher modal loss factor than SED and MSO methods. The numerical model and topology optimization approach were also experimentally validated.

Nevertheless, most of the literature on the layout optimization for CLD treatment has mainly focused on optimization of structural dynamic properties, such as maximizing the modal loss factor ${ }^{9,10}$ and the structural damping ${ }^{11}$ or minimizing the displacement amplitude. ${ }^{12}$ Only a few studies have considered the application of the topology optimization for CLD treatment to acoustical problems. For example, W. Akl et al. attempted to minimize fluid-structure acoustical radiation power in a closed acoustical cavity coupled with a plate by the use of moving asymptotes (MMA). ${ }^{13}$ However, in their study, the objective was not acoustical parameters and the plate was not treated with CLD materials. Liu et al. derived the acoustical sensitivity formula and minimized the sound pressure and sound radiation power by optimizing the layer thickness with a sequential linear programming algorithm. ${ }^{14}$ In these studies, acoustical sensitivity was calculated with an optimization process. In fact, the acoustical sensitivity calculation was difficult in the optimization due to the structural and acoustical coupling.

Hence, an acoustical topology optimization approach for the layout of CLD treatments using the genetic algorithm (GA) was developed to minimize sound radiation power induced vibrating of the plate coupled with a closed acoustical cavity. It

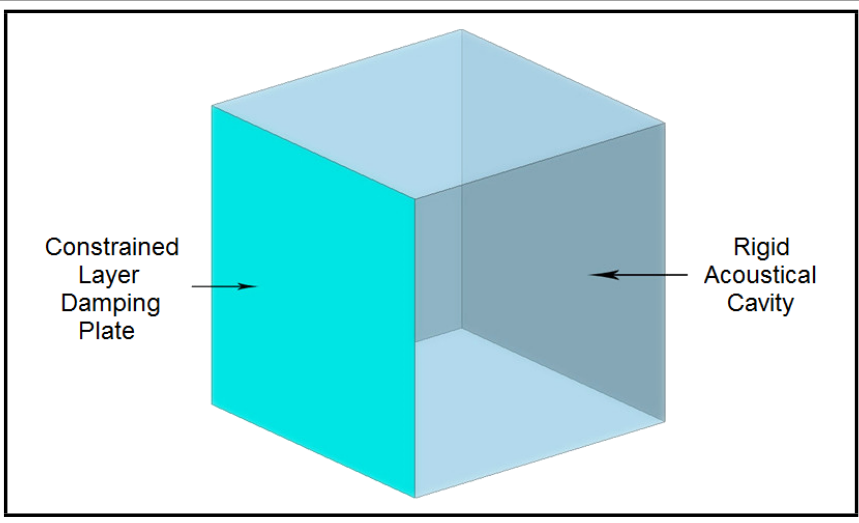

Figure 1. Closed acoustical cavity system.

is worth mentioning that the calculation of acoustical sensitivity in the optimization was avoided. On the other hand, the specific mode frequency was tracked at each optimization iteration. It was based on the fact that during the optimization iteration, change on locations of CLD patches caused a shift in structural natural frequency. Therefore, it was seen that sound radiation power and sound pressure attenuation in the cavity could be attributed only to the acoustical optimization.

This paper is organized in six sections. A brief introduction has been presented in Section 1. A finite element model of CLD/plate and acoustical boundary element analysis for closed acoustical cavity coupled with a flexible plate are developed in Section 2. The formulation of acoustical topology optimization problem is developed and optimization strategy based on GA is presented in Section 3. Numerical example and results are demonstrated in Section 4 and experimental verification is carried out in Section 5. A brief summary is given in Section 6.

\section{ACOUSTICAL RADIATION ANALYSIS FOR THE CLOSED ACOUSTICAL CAVITY}

\subsection{Overview}

A closed acoustical cavity with a flexible plate is shown in Fig. 1. A rectangular flexible plate was coupled with an acoustical cavity, which had five rigid walls. The plate was subjected to external excitation and a finite element model was developed to predict vibration velocity on the flexible plate surface. Then, vibration velocity was considered as a boundary condition of the cavity and the boundary element method (BEM) was used to predict sound radiation from the plate in the cavity. Moreover, a constrained layer damping (CLD) treatment was pasted out on the flexible plate to reduce internal sound radiation.

\subsection{Finite Element Model of CLD/Plate}

The finite element of CLD/plate is illustrated in Fig. 2. It is a 4-node quad element with 7 degrees of freedom per node $\left(u_{c}\right.$, $v_{c}, u_{p}, v_{p}, w, \theta_{x}$, and $\theta_{y}$ ), representing displacements in the $x$-direction and $y$-direction of the constrained and base plate layers, the transverse displacement of the node, and rotations about $x$-axis and $y$-axis, respectively. $h_{p}, h_{v}$, and $h_{c}$ are the thickness of the base layer, the damping layer, and the constrained layer, respectively; $u_{p}$ and $v_{p}$ are displacements in the $x$-direction and the $y$-direction of the base layer; $u_{c}$ and $v_{c}$ are displacements in the $x$-direction and the $y$-direction of the constrained layer; $w$ is the transverse displacement of the node; $\theta_{x}$ 


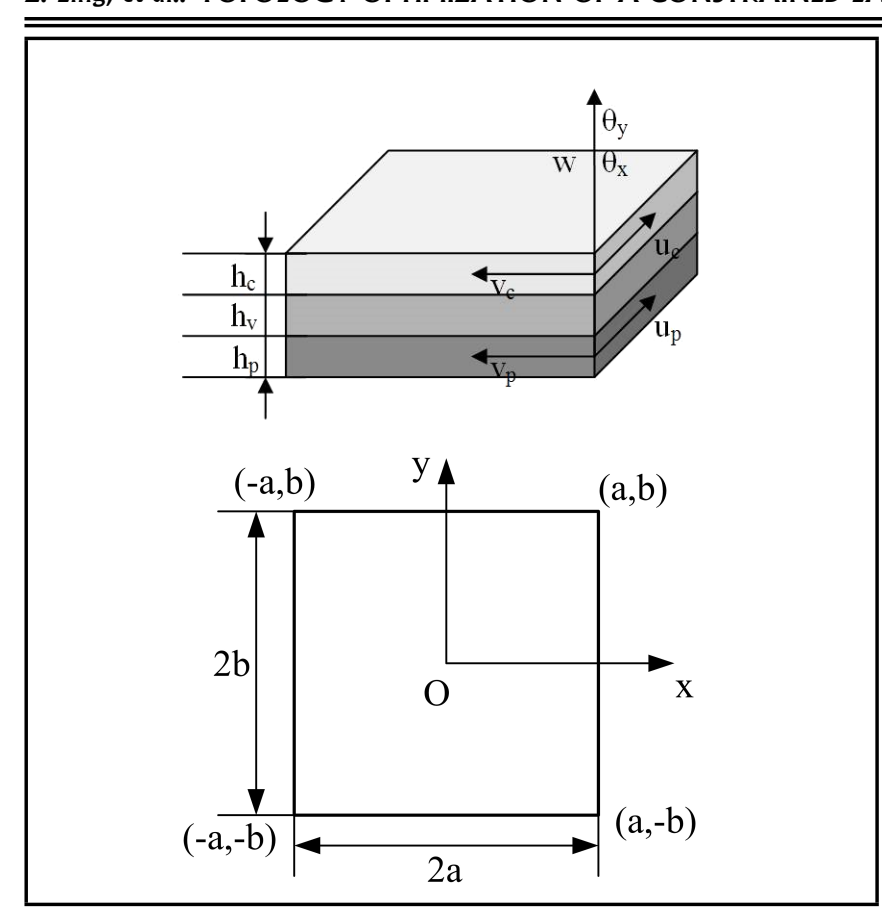

Figure 2. The finite element for the CLD treatment plate.

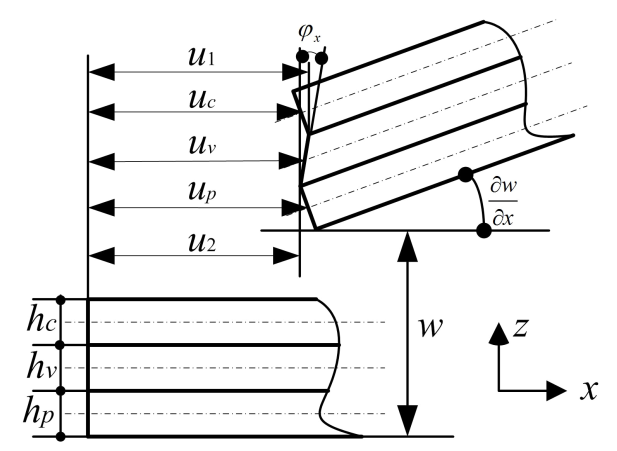

Figure 3. The movement relationship.

and $\theta_{y}$ are rotations about the $x$-axis and the $y$-axis; and $a$ and $b$ are half of the element length.

In the finite element model, it was assumed that transverse displacements $w$ at any point on the same cross section of $\mathrm{CLD} /$ plate were equal. The constrained layer and the base plate layer were assumed to be elastic, dissipate no energy, and their shear strains were negligible. In addition, the damping material was assumed to be linear visco-elastic and all the layers were considered to be bonded together perfectly.

The movement relationship of each layer of the finite element is shown in Fig. 3. The shear deformation and displacements in the $x$-direction and $y$-direction of the damping layer can be derived as follows:

$$
\begin{aligned}
& \beta_{x}=\frac{u_{c}-u_{p}}{h_{v}}+\frac{d}{h_{v}} \frac{\partial w}{\partial x} \\
& \beta_{y}=\frac{u_{c}-u_{p}}{h_{v}}+\frac{d}{h_{v}} \frac{\partial w}{\partial y} ; \\
& u_{v}=\frac{1}{2}\left(u_{c}+u_{p}+\frac{h_{c}-h_{p}}{2} \frac{\partial w}{\partial x}\right) ; \\
& v_{v}=\frac{1}{2}\left(v_{c}+v_{p}+\frac{h_{c}-h_{p}}{2} \frac{\partial w}{\partial y}\right) ;
\end{aligned}
$$

where $d=\left(h_{c}+h_{p}\right) / 2+h_{v}$ is the distance from the plate's neutral surface to the constrained layer's neutral surface. The subscript $v$ denotes the damping layer.

The nodal displacement vector, composed of seven degrees of freedom per node as mentioned above, is given by:

$$
\boldsymbol{\delta}^{(e)}=\left\{u_{c i} v_{c i} u_{p i} v_{p i} w \theta_{x i} \theta_{y i}\right\}^{T}, \quad i=1,2,3,4 .
$$

Therefore, the displacements at any location inside the element can be determined from:

$$
\left\{u_{c} v_{c} u_{p} v_{p} w \theta_{x} \theta_{y}\right\}^{T}=\mathbf{N} \boldsymbol{\delta}^{(e)}
$$

where $\mathbf{N}=\left\{\begin{array}{lllllll}\mathbf{N}_{u c} & \mathbf{N}_{v c} & \mathbf{N}_{u p} & \mathbf{N}_{v p} & \mathbf{N}_{w} & \mathbf{N}_{w, x} & \mathbf{N}_{w, y}\end{array}\right\}^{T}$ is the shape function matrix. $\mathbf{N}_{u c}, \mathbf{N}_{v c}, \mathbf{N}_{u p}, \mathbf{N}_{v p}, \mathbf{N}_{w}, \mathbf{N}_{w, x}$, $\mathbf{N}_{w, y}$ are shape functions corresponding to $u_{c}, v_{c}, u_{p}, v_{p}, w$, $\theta_{x}$, and $\theta_{y}$, respectively.

In addition, shape functions corresponding to the displacement and shear deformation of the damping layer can be yielded as:

$$
\begin{aligned}
\mathbf{N}_{u v} & =\frac{1}{2}\left(\mathbf{N}_{u c}+\mathbf{N}_{u p}+\frac{h_{c}-h_{p}}{2} \mathbf{N}_{w, x}\right) \\
\mathbf{N}_{v v} & =\frac{1}{2}\left(\mathbf{N}_{v c}+\mathbf{N}_{v p}+\frac{h_{c}-h_{p}}{2} \mathbf{N}_{w, y}\right) \\
\mathbf{N}_{\beta x v} & =\frac{1}{h_{v}}\left[\mathbf{N}_{u c}-\mathbf{N}_{u p}+\left(\frac{h_{c}+h_{p}}{2}+h_{v}\right) \mathbf{N}_{w, x}\right] \\
\mathbf{N}_{\beta y v} & =\frac{1}{h_{v}}\left[\mathbf{N}_{u c}-\mathbf{N}_{u p}+\left(\frac{h_{c}+h_{p}}{2}+h_{v}\right) \mathbf{N}_{w, y}\right]
\end{aligned}
$$

Furthermore, strain-displacement relations and stress-strain relations for each layer are derived as:

$$
\begin{aligned}
\varepsilon_{j x} & =\frac{\partial u_{j}}{\partial x}+z \frac{\partial^{2} w}{\partial x^{2}} \\
\varepsilon_{j y} & =\frac{\partial v_{j}}{\partial y}+z \frac{\partial^{2} w}{\partial y^{2}} \\
\gamma_{j x y} & =\frac{\partial u_{j}}{\partial y}+\frac{\partial v_{j}}{\partial x}+2 z \frac{\partial^{2} w}{\partial x \partial y} \\
\sigma_{j x} & =\frac{E_{j}}{1-\mu_{j}^{2}}\left(\varepsilon_{j x}+\mu_{j} \varepsilon_{j y}\right) \\
\sigma_{j y} & =\frac{E_{j}}{1-\mu_{j}^{2}}\left(\varepsilon_{j y}+\mu_{j} \varepsilon_{j x}\right) \\
\tau_{j x y} & =\frac{E_{j}}{2\left(1-\mu_{j}\right)} \gamma_{j x y}
\end{aligned}
$$

where $j=p, c, v$ denotes the base plate layer, the constrained layer, and the damping layer, respectively.

Furthermore, the dynamic equation of CLD/plate can be derived on the basis of energy approach. The kinetic energies and strain potential energies of the three layers can be expressed as follows:

$$
\begin{aligned}
T_{j}^{e} & =\frac{1}{2} \rho_{j} \iiint_{V}\left[\left(\frac{\partial u_{j}}{\partial t}\right)^{2}+\left(\frac{\partial u_{j}}{\partial t}\right)^{2}+\left(\frac{\partial u_{j}}{\partial t}\right)^{2}\right] d V \\
& =\frac{1}{2} \boldsymbol{\delta}^{(e) T} \rho_{j} h_{j} \int_{-a-b}^{a} \int_{-b}^{b}\left(\mathbf{N}_{u j}^{T} \mathbf{N}_{u j}+\mathbf{N}_{v j}^{T} \mathbf{N}_{v j}+\mathbf{N}_{w}^{T} \mathbf{N}_{w}\right) d x d y \boldsymbol{\delta}^{(e)} \\
& =\frac{1}{2} \boldsymbol{\delta}^{(e) T} \mathbf{M}_{j}^{(e)} \boldsymbol{\delta}^{(e)}, \quad j=p, c, v
\end{aligned}
$$




$$
\begin{aligned}
& \hline E_{j}^{(e)}=\frac{1}{2} \iiint_{V} \varepsilon_{j}^{*} \sigma_{j} d V \\
&=\frac{1}{2} \iiint_{V}\left(\sigma_{j x} \varepsilon_{j x}+\sigma_{j y} \varepsilon_{j y}+\sigma_{j z} \varepsilon_{j z}\right) d V \\
&=\frac{1}{2} \boldsymbol{\delta}^{(e) T}\left(h_{j} \int_{-a}^{a} \int_{-b}^{b} \mathbf{B}_{j}^{T} \mathbf{D}_{j} \mathbf{B}_{j} d x d y+\right. \\
&=\frac{1}{2} \boldsymbol{\delta}^{(e) T} \mathbf{K}_{j}^{e} \boldsymbol{\delta}^{(e)}, \quad j=p, c, v . \\
&\left.\int_{-a}^{a} \int_{-b}^{b} \mathbf{B}_{j}^{T} \mathbf{D}_{j} \mathbf{B}_{j} d x d y\right) \boldsymbol{\delta}^{(e)}
\end{aligned}
$$

The potential energy of the damping layer due to shear deformation can be written as:

$$
\begin{aligned}
E_{\beta v}^{(e)} & =\frac{1}{2} \iiint_{V}\left(G \beta_{x}^{2}+G \beta_{y}^{2}\right) d V \\
& =\frac{1}{2} \boldsymbol{\delta}^{(e) T}\left[G h_{v} \int_{-a-b}^{a b}\left(\mathbf{N}_{\beta x v}^{T} \mathbf{N}_{\beta x v}+\mathbf{N}_{\beta y v}^{T} \mathbf{N}_{\beta y v}\right) d x d y\right] \boldsymbol{\delta}^{(e)} \\
& =\frac{1}{2} \boldsymbol{\delta}^{(e) T} \mathbf{K}_{\beta v}^{(e)} \boldsymbol{\delta}^{(e)}
\end{aligned}
$$

Finally, the mass matrices and stiffness matrices of the element can be generated as:

$$
\begin{aligned}
& \mathbf{M}^{(e)}=\mathbf{M}_{p}^{(e)}+\mathbf{M}_{c}^{(e)}+\mathbf{M}_{v}^{(e)} \\
& \mathbf{K}^{(e)}=\mathbf{K}_{p}^{(e)}+\mathbf{K}_{c}^{(e)}+\mathbf{K}_{v}^{(e)}+\mathbf{K}_{\beta v}^{(e)} .
\end{aligned}
$$

The global mass and stiffness matrices of the CLD plate are thus obtained:

$$
\begin{aligned}
& \mathbf{M}=\sum_{e=1}^{n} \mathbf{M}^{(e)} \\
& \mathbf{K}=\sum_{e=1}^{n} \mathbf{K}^{(e)} .
\end{aligned}
$$

Finally, the dynamic equation of CLD/plate is given as:

$$
\mathbf{M X}+\mathbf{K X}=\mathbf{F} .
$$

The vibration velocity on the surface of the CLD plate can be obtained by Eq. (24), and it was considered as a boundary condition of the cavity in the sound radiation analysis.

\subsection{Acoustical Boundary Element Analysis}

The Helmholtz acoustical boundary integral equation for interior acoustical problem is given as follows ${ }^{15}$ :

$C(\alpha) p(\alpha)=\frac{1}{2 \pi} \int_{S} \frac{\partial p(\xi)}{\partial n} G(\alpha, \xi) d S-\frac{1}{2 \pi} \int_{S} p(\xi) \frac{\partial G(\alpha, \xi)}{\partial n} d S ;$

where $G(\alpha, \xi)$ is Green's function, $C$ is a constant that depends on the location of point $\alpha, \xi$ is a point on acoustical field boundary, $\alpha$ is the field point, $p$ is the sound pressure, and $\partial / \partial n$ is the derivative related to a normal vector defined on the

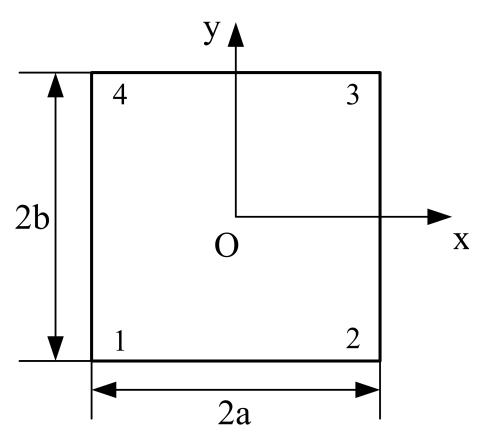

Figure 4. The boundary element.

boundary surface $S$. The explicit expression for the Green's function is:

$$
G(\alpha, \xi)=\frac{e^{-i k r}}{r} ;
$$

where $r=|\alpha-\xi|$ is the distance between two points $\alpha$ and $\xi$, and $k$ is the wave number, which is the ratio of angular frequency $\omega$ to sound speed $c$. The coefficient $C$ of Eq. (25) is expressed as:

$$
C(\alpha)= \begin{cases}2 & \text { when } \alpha \text { is inside the field } \\ 1 & \text { when } \alpha \text { is on the boundary } \\ 0 & \text { when } \alpha \text { is outside the field }\end{cases}
$$

To solve Eq. (25) numerically, the boundary surface $S$ was divided into a number of boundary elements. The boundary element is shown schematically in Fig. 4. The number of elements and nodes are denoted by $N$ and $L$, respectively. For each position of $Q$, the boundary integral in Eq. (25) can be replaced by a sum of integrals over the elements.

The coordinates $\left(x_{Q}, y_{Q}, z_{Q}\right)$, sound pressure $p_{Q}$, and vibration velocity $v_{Q}$ at any point $Q$ on an element are all assumed to be related to the nodal values by Eq. (28) to Eq. (30):

$$
\begin{aligned}
x_{Q}=\sum_{i=1}^{4} N_{i} x_{i}, \quad y_{Q} & =\sum_{i=1}^{4} N_{i} y_{i}, \quad z_{Q}=\sum_{i=1}^{4} N_{i} z_{i} \\
p_{Q} & =\mathbf{N p}=\sum_{i=1}^{4} N_{i} p_{i} \\
v_{Q} & =\mathbf{N v}=\sum_{i=1}^{4} N_{i} v_{i}
\end{aligned}
$$

in which $N_{i}$ is the shape function of the node $i$ in an element:

$$
N_{i}=\frac{1}{4}\left(1+\frac{x}{x_{i}}\right)\left(1+\frac{y}{y_{i}}\right), \quad i=1,2,3,4 .
$$

When $\alpha$ is on the boundary surface $S$, the sound pressure at point $\alpha$ can be calculated as follows:

$$
p(\alpha)=\frac{1}{2 \pi} \int_{S} \frac{\partial p(\xi)}{\partial n} G(\alpha, \xi) d S-\frac{1}{2 \pi} \int_{S} p(\xi) \frac{\partial G(\alpha, \xi)}{\partial n} d S .
$$

For simplicity, $S_{1}, S_{2}, S_{3}, S_{4}, S_{5}$, and $S_{6}$ denote the boundary surfaces ABCD, EFGH, BCGF, AEHD, ABFE, and DCGH, as shown in Fig. 1. Therefore, $S$ is equal to the sum of $S_{i}(i=1,2, \ldots 6)$, that is, $S=S_{1}+S_{2}+S_{3}+S_{4}+S_{5}+S_{6}$. 
For a closed acoustical cavity in Fig. 1, acoustical boundary condition is as follows:

$$
\frac{\partial p}{\partial n}= \begin{cases}0 & \text { on the rigid surface } \\ -i \omega \rho v & \text { on the flexible surface }\end{cases}
$$

where $\rho$ is air density, $v$ is normal vibration velocity and $i^{2}=$ -1. Substituting Eq. (33) into Eq. (32) results in:

$$
\begin{aligned}
p(\alpha)= & \frac{1}{2 \pi} \int_{S_{1}}(-i \rho \omega v(\xi)) G(\alpha, \xi) d S-\frac{1}{2 \pi} \int_{S_{1}} p(\xi) \frac{\partial G(\alpha, \xi)}{\partial n} d S \\
& -\frac{1}{2 \pi} \int_{S_{2}} p(\xi) \frac{\partial G(\alpha, \xi)}{\partial n} d S-\frac{1}{2 \pi} \int_{S_{3}} p(\xi) \frac{\partial G(\alpha, \xi)}{\partial n} d S \\
& -\frac{1}{2 \pi} \int_{S_{4}} p(\xi) \frac{\partial G(\alpha, \xi)}{\partial n} d S-\frac{1}{2 \pi} \int_{S_{5}} p(\xi) \frac{\partial G(\alpha, \xi)}{\partial n} d S \\
& -\frac{1}{2 \pi} \int_{S_{6}} p(\xi) \frac{\partial G(\alpha, \xi)}{\partial n} d S .
\end{aligned}
$$

Meanwhile, the derivative of Green's function $G$ can be given as:

$$
\frac{\partial G(\alpha, \xi)}{\partial n}=-\frac{i k r+1}{r^{2}} e^{-i k r} \frac{\partial r}{\partial n}=G^{\prime} \frac{\partial r}{\partial n}
$$

where,

$$
\frac{\partial r}{\partial n}=\left\{\begin{array}{rc}
-\left|y_{\alpha}-y_{\xi}\right| & \text { the point } \xi \text { is on the surface } S_{1} \\
\left|y_{\alpha}-y_{\xi}\right| & \text { the point } \xi \text { is on the surface } S_{2} \\
\left|x_{\alpha}-x_{\xi}\right| & \text { the point } \xi \text { is on the surface } S_{3} \\
-\left|x_{\alpha}-x_{\xi}\right| & \text { the point } \xi \text { is on the surface } S_{4} \\
-\left|z_{\alpha}-z_{\xi}\right| & \text { the point } \xi \text { is on the surface } S_{5} \\
\left|z_{\alpha}-z_{\xi}\right| & \text { the point } \xi \text { is on the surface } S_{6}
\end{array}\right.
$$

in which $x_{\alpha}, y_{\alpha}, z_{\alpha}, x_{\xi}, y_{\xi}, z_{\xi}$ are the coordinates of point $\alpha$ and $\xi$. Hence,

$$
\begin{aligned}
p(\alpha)= & \frac{1}{2 \pi} \int_{S_{1}}(-i \rho \omega v(\xi)) G(\alpha, \xi) d S+\frac{1}{2 \pi} \int_{S_{1}} p(\xi) G^{\prime}\left|y_{\alpha}-y_{\xi}\right| d S \\
& -\frac{1}{2 \pi} \int_{S_{2}} p(\xi) G^{\prime}\left|y_{\alpha}-y_{\xi}\right| d S-\frac{1}{2 \pi} \int_{S_{3}} p(\xi) G^{\prime}\left|x_{\alpha}-x_{\xi}\right| d S \\
& +\frac{1}{2 \pi} \int_{S_{4}} p(\xi) G^{\prime}\left|x_{\alpha}-x_{\xi}\right| d S+\frac{1}{2 \pi} \int_{S_{5}} p(\xi) G^{\prime}\left|z_{\alpha}-z_{\xi}\right| d S \\
& -\frac{1}{2 \pi} \int_{S_{6}} p(\xi) G^{\prime}\left|z_{\alpha}-z_{\xi}\right| d S .
\end{aligned}
$$

By replacing Eq. (37) with a sum of integrals over the elements and assuming that point $\alpha$ is node $j$ of one boundary element, then Eq. (37) can be expressed as:

$$
\begin{aligned}
p(j)= & \sum_{m_{1}=1}^{N} \sum_{n=1}^{4} \frac{1}{2 \pi} \int_{\Delta S_{1}}(-i \rho \omega) G(j, \xi) N_{n} d_{\Delta S} v_{m_{1} n} \\
& +\sum_{m_{1}=1}^{N} \sum_{n=1}^{4} \frac{1}{2 \pi} \int_{\Delta S_{1}} N_{n} G^{\prime}\left|y_{j}-y_{\xi}\right| d_{\Delta S} p_{m_{1} n} \\
& -\sum_{m_{2}=1}^{N} \sum_{n=1}^{4} \frac{1}{2 \pi} \int_{\Delta S_{2}} N_{n} G^{\prime}\left|y_{j}-y_{\xi}\right| d_{\Delta S} p_{m_{2} n} \\
& -\sum_{m_{3}=1}^{N} \sum_{n=1}^{4} \frac{1}{2 \pi} \int_{\Delta S_{3}} N_{n} G^{\prime}\left|x_{j}-x_{\xi}\right| d_{\Delta S} p_{m_{3} n} \\
& +\sum_{m_{4}=1}^{N} \sum_{n=1}^{4} \frac{1}{2 \pi} \int_{\Delta S_{4}} N_{n} G^{\prime}\left|x_{j}-x_{\xi}\right| d_{\Delta S} p_{m_{4} n} \\
& +\sum_{m_{5}=1}^{N} \sum_{n=1}^{4} \frac{1}{2 \pi} \int_{\Delta S_{5}} N_{n} G^{\prime}\left|z_{j}-z_{\xi}\right| d_{\Delta S} p_{m_{5} n} \\
& -\sum_{m_{6}=1}^{N} \sum_{n=1}^{4} \frac{1}{2 \pi} \int_{\Delta S_{6}} N_{n} G^{\prime}\left|z_{j}-z_{\xi}\right| d_{\Delta S} p_{m_{6} n} ;
\end{aligned}
$$

where $v_{m n}$ denotes normal vibration velocity at node $n$ of element $m, p_{m n}$ denotes sound pressure at node $n$ of element $m$.

In Eq. (38), the distance between node $j$ and point $\xi$ on element $m$ is simplified to the distance between node $j$ and the centre of element $m$, so that $G(j, \xi)$ and $G^{\prime}(j, \xi)$ are fixed values on element $m$ and the distance between node $j$ and point $\xi$ will never be zero. In addition, $\int_{\Delta S} N_{n} d_{\Delta S}=a b$.

For simplicity, the following parameters are introduced:

$$
b_{j m n}=\frac{1}{2 \pi} \int_{\Delta S_{1}}(-i \rho \omega) G(j, \xi) N_{n} d_{\Delta S}=\frac{-i \rho \omega}{2 \pi} G(j, m) a b
$$

$$
h_{j m n}=\left\{\begin{array}{r}
\frac{1}{2 \pi} \int_{\Delta S_{1}} G^{\prime} N_{n}\left|y_{j}-y_{\xi}\right| d_{\Delta S}=\frac{1}{2 \pi} G^{\prime}\left|y_{j}-y_{\xi}\right| a b \\
\text { the point } \xi \text { in on the surface } S_{1} \\
-\frac{1}{2 \pi} \int_{\Delta S_{2}} G^{\prime} N_{n}\left|y_{j}-y_{\xi}\right| d_{\Delta S}=-\frac{1}{2 \pi} G^{\prime}\left|y_{j}-y_{\xi}\right| a b \\
\text { the point } \xi \text { in on the surface } S_{2} \\
-\frac{1}{2 \pi} \int_{\Delta S_{3}} G^{\prime} N_{n}\left|x_{j}-x_{\xi}\right| d_{\Delta S}=-\frac{1}{2 \pi} G^{\prime}\left|x_{j}-x_{\xi}\right| a b \\
\text { the point } \xi \text { in on the surface } S_{3} \\
\frac{1}{2 \pi} \int_{\Delta S_{4}} G^{\prime} N_{n}\left|x_{j}-x_{\xi}\right| d_{\Delta S}=\frac{1}{2 \pi} G^{\prime}\left|x_{j}-x_{\xi}\right| a b \\
\text { the point } \xi \text { in on the surface } S_{4} \\
\frac{1}{2 \pi} \int_{\Delta S_{5}} G^{\prime} N_{n}\left|z_{j}-z_{\xi}\right| d_{\Delta S}=\frac{1}{2 \pi} G^{\prime}\left|z_{j}-z_{\xi}\right| a b \\
\text { the point } \xi \text { in on the surface } S_{5} \\
-\frac{1}{2 \pi} \int_{\Delta S_{6}} G^{\prime} N_{n}\left|z_{j}-z_{\xi}\right| d_{\Delta S}=\frac{1}{2 \pi} G^{\prime}\left|z_{j}-z_{\xi}\right| a b \\
\text { the point } \xi \text { in on the surface } S_{6}
\end{array}\right.
$$


Here, the formula of sound pressure at node $j$ can be simplified to:

$$
\begin{aligned}
p(j)= & \sum_{m_{1}=1}^{N} \sum_{n=1}^{4} b_{j m_{1} n} v_{m_{1} n}+\sum_{m_{1}=1}^{N} \sum_{n=1}^{4} h_{j m_{1} n}^{S_{1}} p_{m_{1} n} \\
& -\sum_{m_{2}=1}^{N} \sum_{n=1}^{4} h_{j m_{2} n}^{S_{2}} p_{m_{2} n}-\sum_{m_{3}=1}^{N} \sum_{n=1}^{4} h_{j m_{3} n}^{S_{3}} p_{m_{3} n} \\
& +\sum_{m_{4}=1}^{N} \sum_{n=1}^{4} h_{j m_{4} n}^{S_{4}} p_{m_{4} n}+\sum_{m_{5}=1}^{N} \sum_{n=1}^{4} h_{j m_{5} n}^{S_{5}} p_{m_{5} n} \\
& -\sum_{m_{6}=1}^{N} \sum_{n=1}^{4} h_{j m_{6} n}^{S_{6}} p_{m_{6} n} \\
= & \mathbf{b}_{j} \mathbf{V}+\mathbf{h}_{j} \mathbf{P} .
\end{aligned}
$$

Similarly, sound pressure at all other nodes on the boundary surface can be calculated by Eq. (41), then a set of equations is obtained as:

$$
\mathbf{P}=\mathbf{B V}+\mathbf{H P} .
$$

The nodal sound pressure on the boundary surface are given as:

$$
\mathbf{P}=(\mathbf{I}-\mathbf{H})^{-1} \mathbf{B V}
$$

where $\mathbf{P}$ is the nodal sound pressure vector on the boundary surface $S, \mathbf{V}$ is the nodal normal vibration velocity vector on the boundary surface $S_{1}$ (surface $\mathrm{ABCD}$ ), and $\mathbf{H}$ and $\mathbf{B}$ are the coefficient matrices.

The structural sound radiation power can be calculated from sound pressure and vibration velocity on the surface:

$$
W=\frac{1}{2} \int_{S} \operatorname{Re}\left[\mathbf{v}^{*}(Q) p(Q)\right] d_{S}
$$

where $Q$ is a point on surface of the structure, $\mathbf{v}^{*}(Q)$ is a complex conjugate of normal vibration velocity at point $Q$ on the surface, $p(Q)$ is the sound pressure at point $Q$, and Re means the real part.

For the closed acoustical cavity, sound radiation power of the flexible plate can be replaced by the sum of sound radiation power radiated from $\mathrm{N}$ boundary elements:

$$
\begin{aligned}
W & =\frac{1}{2} \int_{S_{1}} \operatorname{Re}\left[v^{*}(P) p(P)\right] d_{S(P)} \\
& =\sum_{m=1}^{N} \frac{1}{2} \int_{\Delta S} \operatorname{Re}\left[v_{m}^{*}(P) p_{m}(P)\right] d_{\Delta S(P)} \\
& =\operatorname{Re} \sum_{m=1}^{N} \frac{1}{2} \int_{\Delta S}\left[\mathbf{v}_{m}^{*} \mathbf{N}^{T} \mathbf{N} \mathbf{p}_{m}\right] d_{\Delta S(P)} \\
& =\operatorname{Re} \sum_{m=1}^{N} \mathbf{v}_{m}^{*} \frac{1}{2} \int_{\Delta S} \mathbf{N}^{T} \mathbf{N} d_{\Delta S(P)} p_{m} \\
& =\operatorname{Re} \sum_{m=1}^{N} \mathbf{v}_{m}^{*} \mathbf{D} \mathbf{p}_{m} ; \\
\mathbf{D} & =\frac{1}{2} \int_{\Delta S} \mathbf{N}^{T} \mathbf{N} d_{\Delta S}=\frac{1}{18} a b\left[\begin{array}{llll}
4 & 2 & 1 & 2 \\
2 & 4 & 2 & 1 \\
1 & 2 & 4 & 2 \\
2 & 1 & 2 & 4
\end{array}\right] ;
\end{aligned}
$$

\begin{tabular}{|c|c|c|c|c|c|}
\hline 31 & 32 & 33 & 34 & 35 & 36 \\
\hline 25 & 26 & 27 & 28 & 29 & 30 \\
\hline 19 & 20 & 21 & 22 & 23 & 24 \\
\hline 13 & 14 & 15 & 16 & 17 & 18 \\
\hline 7 & 8 & 9 & 10 & 11 & 12 \\
\hline 1 & 2 & 3 & 4 & 5 & 6 \\
\hline
\end{tabular}

Figure 5. The coding schematic diagram.

where $\mathbf{p}_{m}$ is the nodal sound pressure vector of element $m, \mathbf{v}_{m}^{*}$ is the complex conjugate of nodal normal vibration velocity vector of element $m$. However, it was noted that $\mathbf{p}_{m}$ and $\mathbf{v}_{m}^{*}$ were just only nodal values on surface $S_{1}$ (flexible plate).

On the other hand, when point $\alpha$ is inside the acoustical field, the Helmholtz acoustical boundary integral equation can be written as:

$$
2 p(\alpha)=\frac{1}{2 \pi} \int_{S} \frac{\partial p(\xi)}{\partial n} G(\alpha, \xi) d S-\frac{1}{2 \pi} \int_{S} p(\xi) \frac{\partial G(\alpha, \xi)}{\partial n} d S .
$$

So sound pressure at point $\alpha$ can be calculated as follows:

$$
p(\alpha)=\mathbf{b V}+\mathbf{h P}
$$

where $\mathbf{V}$ is the nodal normal vibration velocity vector on boundary surface $S_{1}$ (surface ABCD). $\mathbf{P}$ is the nodal sound pressure vector on the whole boundary surface $S$, which can be obtained by Eq. (43). $\mathbf{b}$ and $\mathbf{h}$ are the coefficient matrices.

\section{ACOUSTICAL TOPOLOGY OPTIMIZATION FOR CONSTRAINED LAYER DAMPING}

\subsection{Acoustical Topology Optimization Model}

The acoustical topology optimization model for CLD treatment plate can be described as follows:

$$
\begin{array}{cc}
\text { Find } & X=\left\{\begin{array}{llll}
x_{1} & x_{2} & \ldots & x_{n}
\end{array}\right\} \\
\text { Min } & W \\
\text { s.t. } & x_{\min } \leq x_{i} \leq x_{\max } \\
& V_{f}=V_{0}
\end{array}
$$

where $X$ is design variable vector, $x_{i}$ represents locations of CLD material on the plate, and $n$ is the number of design variables. $W$ denotes sound radiation power and the objective function can be explained literally as to minimize sound radiation power. $x_{\min }, x_{\max }$ denote the limits of design variables. $V_{f}$ is the constraint of material consumption and $V_{0}$ is the actual consumption of CLD material in the optimization.

\subsection{Optimization Strategy}

In this paper, a genetic algorithm with an elitist strategy was used to solve the acoustical topology optimization problem. The concrete steps for the algorithm are given as follows: 


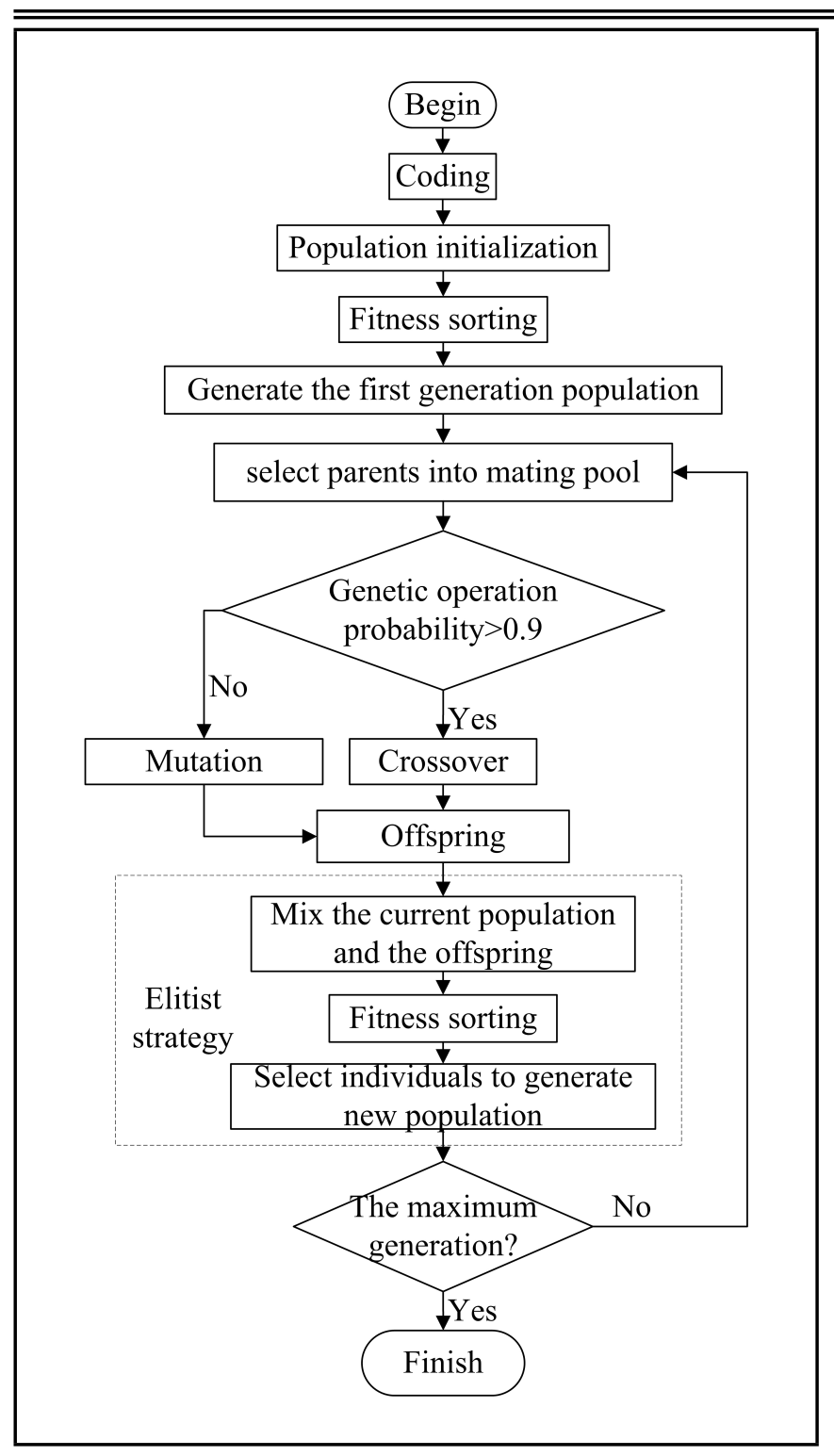

Figure 6. The program flowchart for GA.

\section{Coding}

Firstly, one design variable vector $X$ need to be encoded to form an individual by some coding method. In this paper, the integer coding method is used to encode the design variable vector $X$ due to the locations of the CLD material. For example, if the plate is divided into 36 elements, as shown in Fig. 5, there are 36 positions for CLD materials. It was assumed that one individual who was encoded in genetic population, is [ 2102531 ]. That means there are CLD materials on the element 2, 10, 25, and 31, respectively.

\section{Population initialization}

The population is initialized using random numbers. If population size in genetic algorithm is $N_{\text {pop }}$, then the initialization size is set to $2 N_{\text {pop }}$.

\section{Fitness sorting}

The individuals are sorted according to the fitness values. The fitness function is expressed as:

$$
f=W .
$$

Here, $W$ is the sound radiation power from the flexible plate.

\section{Select the first generation population}

Select the first $N_{\text {pop }}$ individuals from the sorted individuals as the first generation population.

\section{Select the parents}

The parents were selected by using a binary tournament selection and the size of mating pool was set to be $N_{\text {pop }} / 2$.

6. Generate a random number $r_{p}$ between 0 and 1 If $r_{p}>0.9$, two individuals were selected from the mating pool to take the crossover operation, otherwise one individual was selected from the mating pool to take mutation operation. That means the crossover probability is $90 \%$ and the mutation probability is $10 \%$.

\section{Crossover}

The Laplace Crossover operator is employed and is given as below:

$$
\begin{aligned}
& x_{1}=\bar{x}_{1}+\beta\left|\bar{x}_{1}-\bar{x}_{2}\right| ; \\
& x_{2}=\bar{x}_{2}+\beta\left|\bar{x}_{1}-\bar{x}_{2}\right| ; \\
& \beta=\left\{\begin{array}{ll}
a-b \log (u) & r \leq 0.5 \\
a+b \log (u) & r>0.5
\end{array} ;\right.
\end{aligned}
$$

where $\bar{x}_{1}$ and $\bar{x}_{2}$ were parents which were selected from the mating pool randomly, $x_{1}$ and $x_{2}$ are the children, $a$ and $b$ are Laplace Crossover factors, $u$ and $r$ are two random numbers between 0 and 1 . It is worth mentioning that the two children generated by crossover need to be truncated to be an integer.

\section{Mutation}

The Power Mutation operator was employed and is given as below:

$$
\begin{aligned}
& x= \begin{cases}\bar{x}-s^{m f}\left(\bar{x}-x^{l}\right) & t<r \\
\bar{x}+s^{m f}\left(x^{u}-\bar{x}\right) & t \geq r\end{cases} \\
& t=\frac{\bar{x}-x^{l}}{x^{u}-\bar{x}}
\end{aligned}
$$

where $\bar{x}$ is the parent and $x$ is the child, $x^{l}$ and $x^{u}$ are the limits of the design variables, $m f$ is the Power Mutationfactor, and $s$ and $r$ are two random numbers between $[0,1]$. The child generated by mutation needs to be truncated in order to be an integer.

\section{Elitist Strategy}

The offspring population was combined with the current generation population and all individuals were sorted according to fitness. Then, selection was performed to produce individuals for the next generation. Since all the previous and current best individuals were added in the population, elitism was ensured.

\section{Determination condition}

If the generations had achieved the maximum number of generations, the evolution was terminated. Then, the last generation population was saved and decoded.

The program flowchart for GA is shown in Fig. 6. 
Table 1. Parameters for the closed acoustic cavity.

\begin{tabular}{|c|c|c|}
\hline \multicolumn{2}{|l|}{ Cavity dimensions } & $30 \mathrm{~cm} \times 30 \mathrm{~cm} \times 30 \mathrm{~cm}$ \\
\hline \multicolumn{2}{|l|}{ Fluid domain } & Air at $25^{\circ} \mathrm{C}$ and $1 \mathrm{~atm}$ \\
\hline Flexible plate thickness & $\begin{array}{l}\text { Base layer (Aluminium) } \\
\text { Damping layer } \\
\text { Constrained layer }\end{array}$ & $\begin{array}{c}0.8 \mathrm{~mm} \\
0.05 \mathrm{~mm} \\
0.13 \mathrm{~mm}\end{array}$ \\
\hline
\end{tabular}

Table 2. Parameters for the CLD treatment plate.

\begin{tabular}{|c|c|c|c|}
\hline & Young's modulus $(\mathrm{Pa})$ & Density $\left(\mathrm{kg} \cdot \mathrm{m}^{-3}\right)$ & Poisson's ratio \\
\hline Base layer (Aluminium) & $7.0 \mathrm{e} 10$ & 2800 & 0.3 \\
Damping layer & $12 \mathrm{e} 7$ & 1200 & 0.495 \\
Constrained layer & $7.0 \mathrm{e} 10$ & 2700 & 0.3 \\
\hline
\end{tabular}

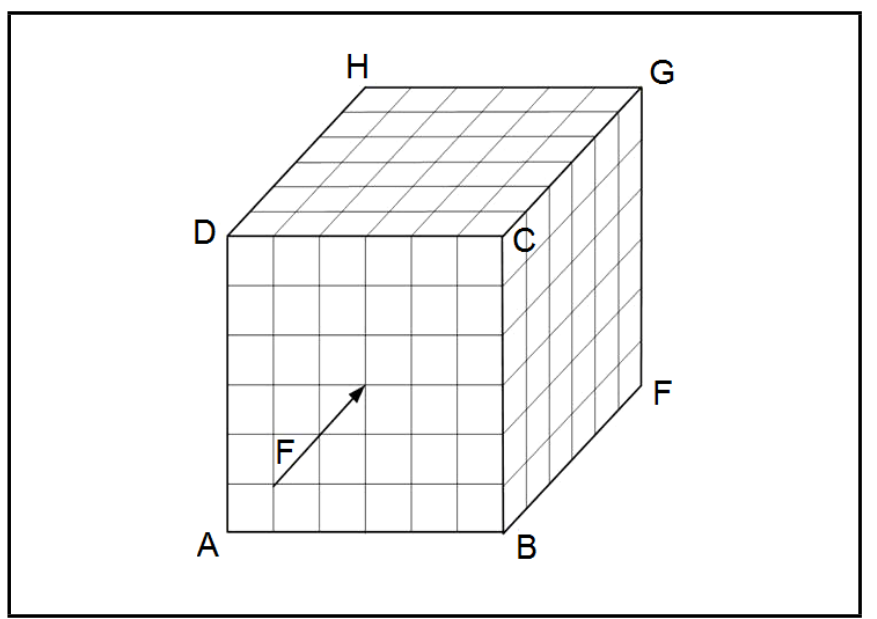

Figure 7. The boundary element model for the closed acoustical cavity.

\section{NUMERICAL RESULTS}

\subsection{Model Setup}

A finite element model for CLD/ plate and a boundary element model for the closed acoustical cavity with a flexible plate, as shown in Fig. 7, was developed. The characteristics of the coupled fluid-structure domains are given in Table 1 and material parameters of CLD/plate are given in Table 2. According to viscoelastic material's Nomogram (SOUNDFOIL 5D401, US), the loss factor can be considered as a constant from $10 \mathrm{~Hz}$ to $1000 \mathrm{~Hz}$. On the other hand, the effect of temperature on the characteristics of viscoelastic material is very small from $20^{\circ} \mathrm{C}$ to $135^{\circ} \mathrm{C}$. Therefore, the shear modulus of the viscoelastic material is described by using complex constant modulus model, $G=G^{\infty}(1+i \eta), \eta=0.85$.

The optimization objective was to minimize the first order of sound radiation power and 2/9, 3/9, 4/9, 5/9, 6/9, 7/9, and $8 / 9$ of material consumption are considered as the constraints, respectively.

An excitation force was applied to the center of flexible plate and it was a unit harmonic force. However, it is worth mentioning that the excitation force was locked to the mode frequency so that the layout of the CLD materials were optimized in such a way as to minimize sound radiation power at that specific modal frequency. More specifically, the first modal frequency was considered as the first odd mode with high acoustical coupling. It was based on the fact that the first odd mode shape of the flexible plate could not be changed when the layout of CLD patches was changed. Hence, the first mode frequency of CLD/plate was calculated again due to the change of CLD (a)

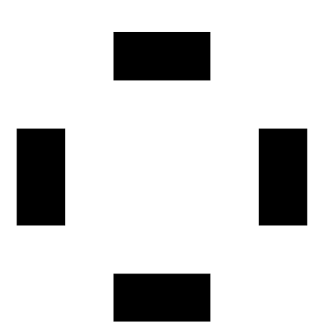

(c)

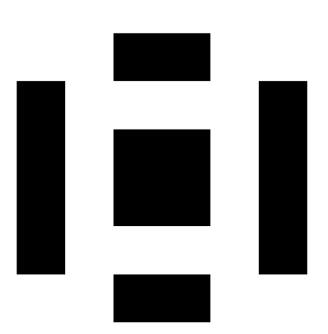

(e)

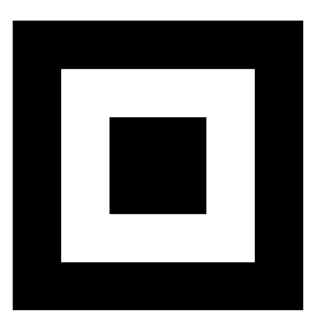

(b)

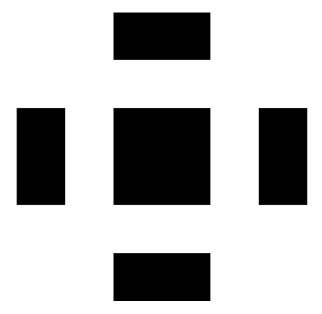

(d)

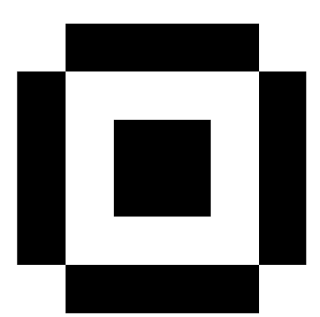

(f)

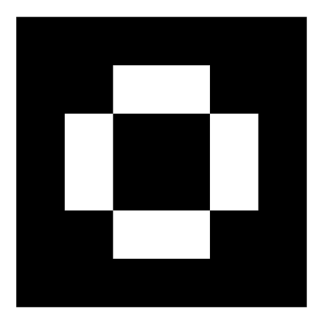

(g)

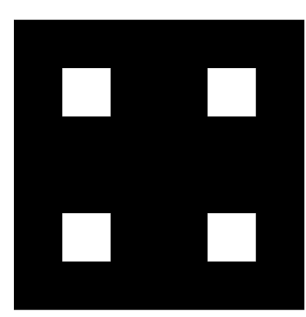

Figure 8. The optimal layout of the constrained layer damping materials (a) $V_{f}=2 / 9$, (b) $V_{f}=3 / 9$, (c) $V_{f}=4 / 9$, (d) $V_{f}=5 / 9$, (e) $V_{f}=6 / 9$, (f) $V_{f}=7 / 9$, and (g) $V_{f}=8 / 9$.

treatments layout and the excitation frequency is locked each at optimization iteration.

In addition, the population size $N_{\text {pop }}$ of genetic algorithm was set to be 100 , and the mating pool size was set to 50 . 


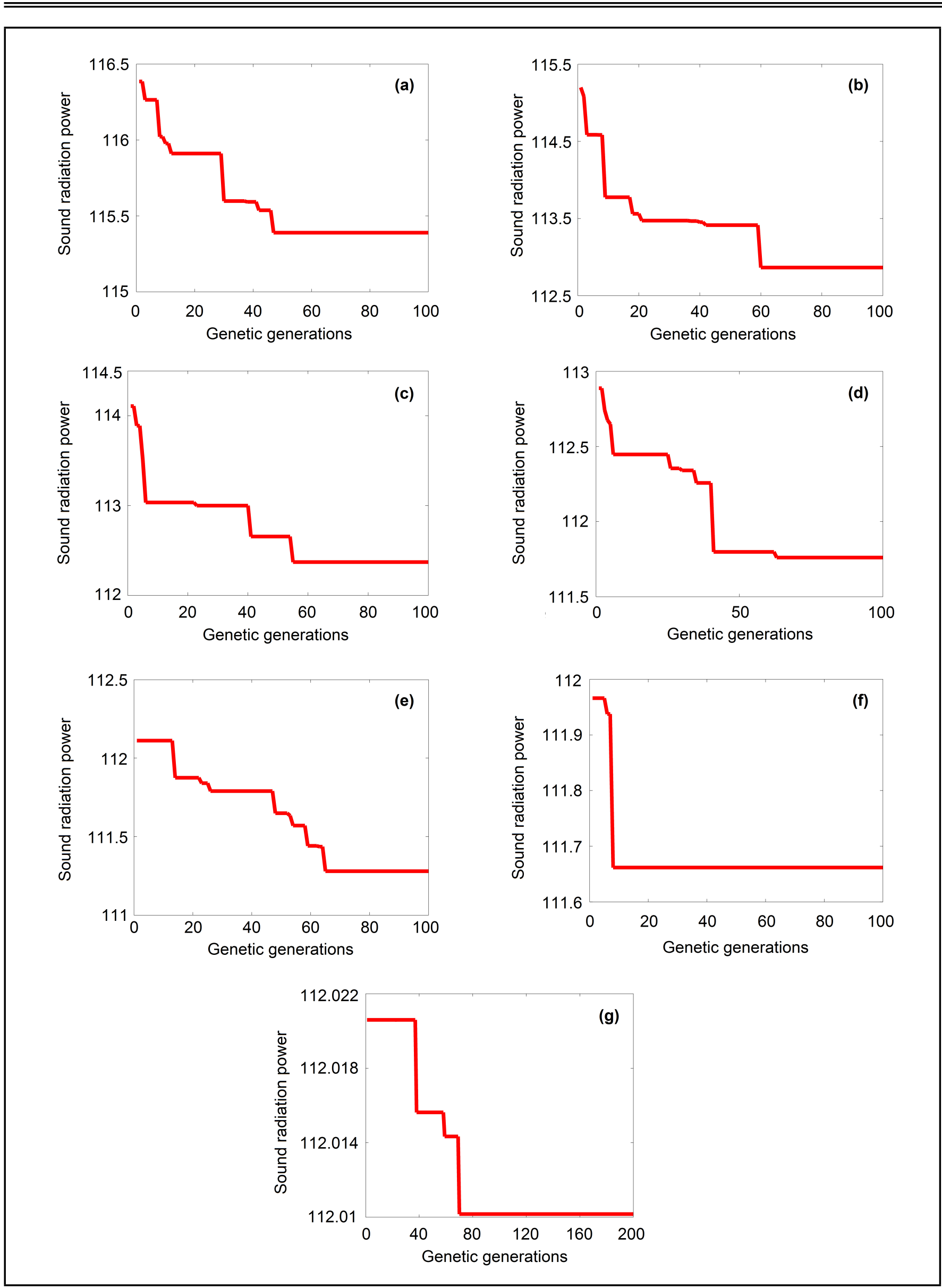

Figure 9. The evolution history of the sound radiation power under different material consumption (a) $V_{f}=2 / 9$, (b) $V_{f}=3 / 9$, (c) $V_{f}=4 / 9$, (d) $V_{f}=5 / 9$, (e) $V_{f}=6 / 9$, (f) $V_{f}=7 / 9$, and (g) $V_{f}=8 / 9$. 


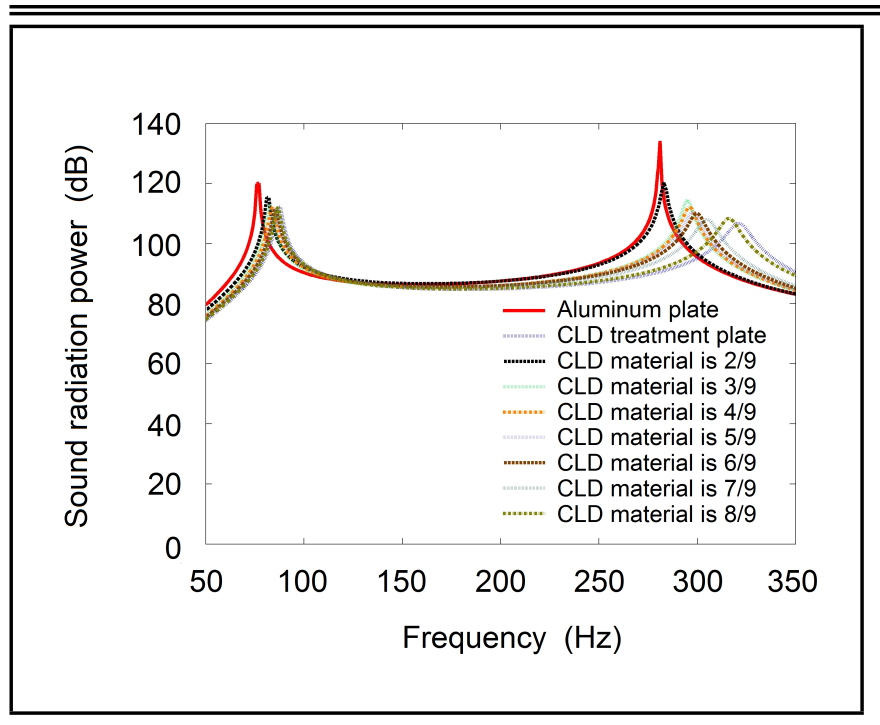

Figure 10. The frequency responses of sound radiation power.

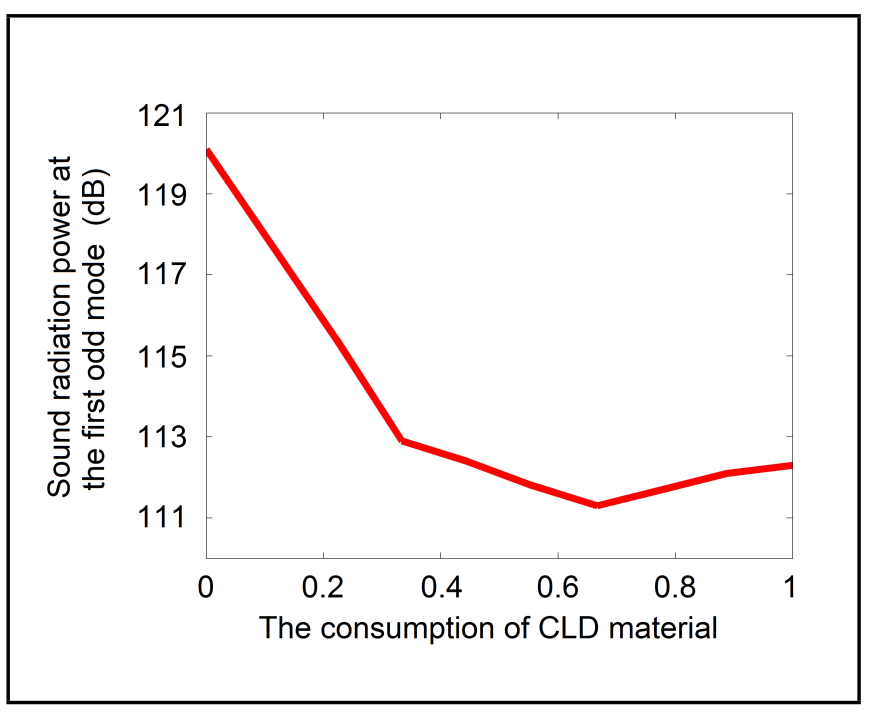

Figure 11. The sound radiation power at the first odd mode.

\subsection{Optimal Layout of Constrained Layer Damping Materials}

The optimal layouts of CLD materials under various material consumptions are shown in Fig. 8. The effect of the optimization process on radiation sound power measured at the first odd mode frequency was monitored. Fig. 9 illustrates solution convergence histories under various material consumptions. Meanwhile, the sound radiation power was aimed at the best individual of each generation population in optimization process.

The frequency response for sound radiation power and sound pressure are investigated. The sound pressure was monitored at the center of the closed acoustical cavity. The frequency responses for optimized CLD/plate under various material consumptions are shown in Fig. 10 and Fig. 12. The frequency responses for the aluminum plate without CLD treatment and the plate with fully CLD treatment are also demonstrated in Fig. 10 and Fig. 12. In addition, Fig. 11 and Fig. 13 illustrate sound radiation power and sound pressure at the first odd mode under various CLD material consumptions.

It is clear that once the optimal layout of CLD treatment

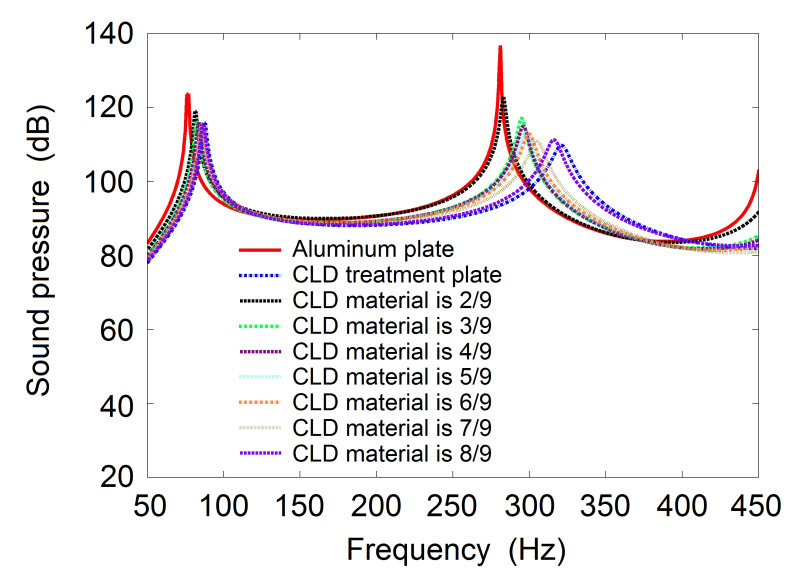

Figure 12. The frequency responses of sound pressure.

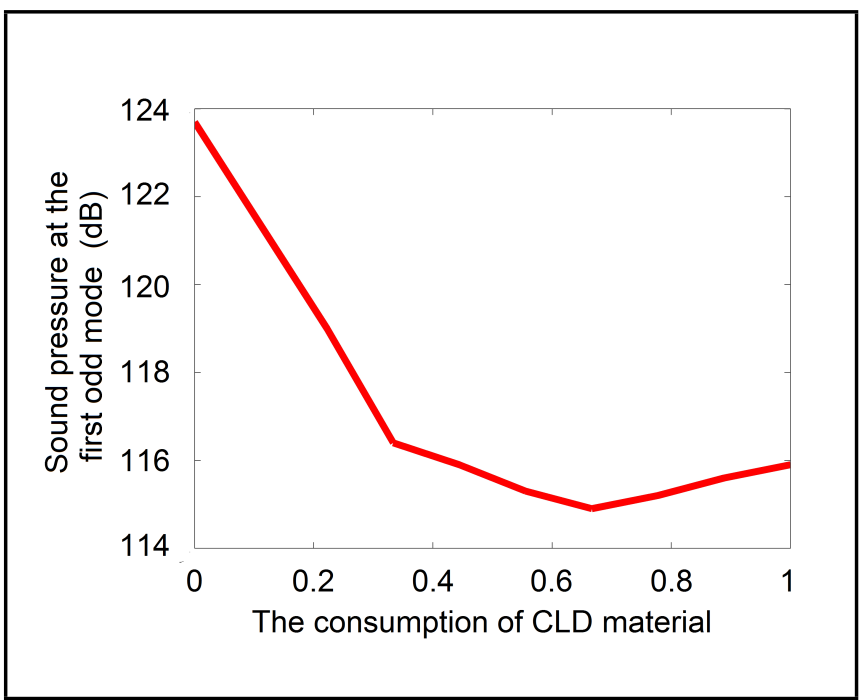

Figure 13. The sound pressure at the first odd mode.

was pasted on the flexible plate in the closed acoustical cavity, radiation sound power and sound pressure inside the cavity reduced significantly. A different optimal layout of CLD treatment could be obtained when the different CLD material consumptions and modal frequencies were considered. This implies that GA, with and elitist strategy method and topology optimization program, was correct and effective. Specifically, when $66.6 \%$ CLD material was applied to the flexible plate in optimal layouts, sound radiation power was attenuated $8.8 \mathrm{~dB}$ compared to the aluminum plate and $1 \mathrm{~dB}$ compared to the fully CLD treatment plate.

\section{EXPERIMENTAL VERIFICATION}

To verify the obtained results experimentally, a set of three different flexible plates were prepared. The first plate was the aluminum plate which has surface dimensions of $30 \mathrm{~cm} \times$ $30 \mathrm{~cm}$ and thickness of $0.8 \mathrm{~mm}$. The second plate was the aluminum plate with full coverage of CLD materials. The third plate was the CLD/plate corresponding to the topology optimization result in 5/9 CLD material consumption. The three flexible plates are shown in Fig. 14. 
A $30 \mathrm{~cm} \times 30 \mathrm{~cm} \times 30 \mathrm{~cm}$ closed acoustical cavity was prepared. The cavity had only one surface coupled to the flexible plate as shown in Fig. 15. Each of the three different plates were mounted and the sound pressure level at the center of the cavity was measured. The plate was mechanically excited at the center with a force hammer and sound pressure level was measured by LMS test system.

Frequency responses for sound pressure inside the acoustical cavity are shown in Fig. 16. The displayed results emphasize effectiveness of topology optimization in attenuating sound pressure level inside the acoustical cavity. Furthermore, the obtained results agree closely with the theoretical predictions displayed in Fig. 12.

\section{CONCLUSIONS}

A finite element model is developed to simulate the vibration of the CLD treatment plate. An acoustical boundary element model for a rigid acoustical cavity coupled with a flexible plate is further developed to predict the sound radiation inside the cavity. An acoustical topology optimization approach based on genetic algorithm is proposed to search the optimal layout of CLD material in the flexible plate. The objective of the optimization is to determine the layout of the CLD material in order to minimize the sound radiation power. The acoustical topology optimization approach is integrated with the finite element model and the boundary element model.

In the optimization, the excitation acting on the plate is locked at the first odd mode to ensure the effectiveness of the optimization in reducing the sound radiation power or the sound pressure at the modal frequency. The analytical model showed considerable attenuation for the first odd mode, as well as the other modes. Especially for the plate with 66.6\% CLD materials, its sound radiation power or the sound pressure is lower than the plate with $100 \%$ CLD materials.

Experimental verification is carried out by manufacturing a topology optimized CLD treatment plate that approximate the optimization result obtained from the analytical model. The plate is coupled to an acoustical cavity. The sound pressure inside the acoustical cavity is measured and compared with the aluminum plate and CLD treatment plate cases. Considerable attenuation in the sound pressure inside the acoustical cavity is obtained and a good match with the analytical model is observed.

This study has shown that the proposed acoustical topology optimization approach can be an effective tool in the design of a wide variety of critical structures, which must be lightweight and operate quietly such as the panels in the vehicle body, aircraft cabin and so on.

\section{ACKNOWLEDGEMENTS}

The research has been made possible by the Natural Science Foundation of China (Grant No. 50775225), the Open Research Foundation of State Key Laboratory of Vehicle NVH and Safety Technology (Grant No. NVHSKL201405), and the Open Research Foundation of Science and Technology on Reliability and Environmental Engineering Laboratory (Grant No.KHZS20153001). (a)

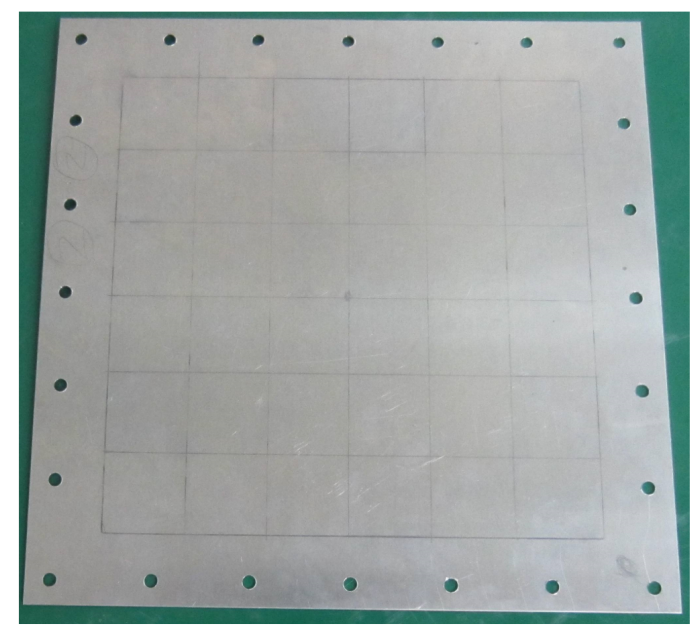

(b)

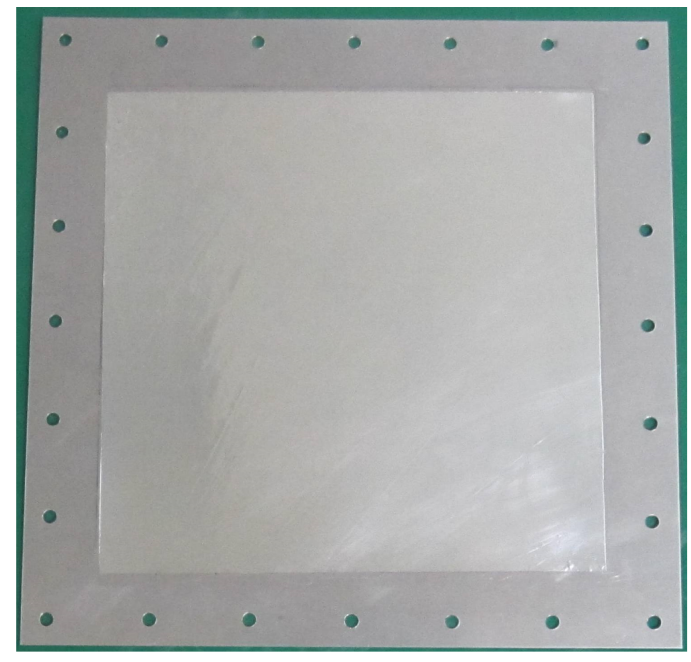

(c)

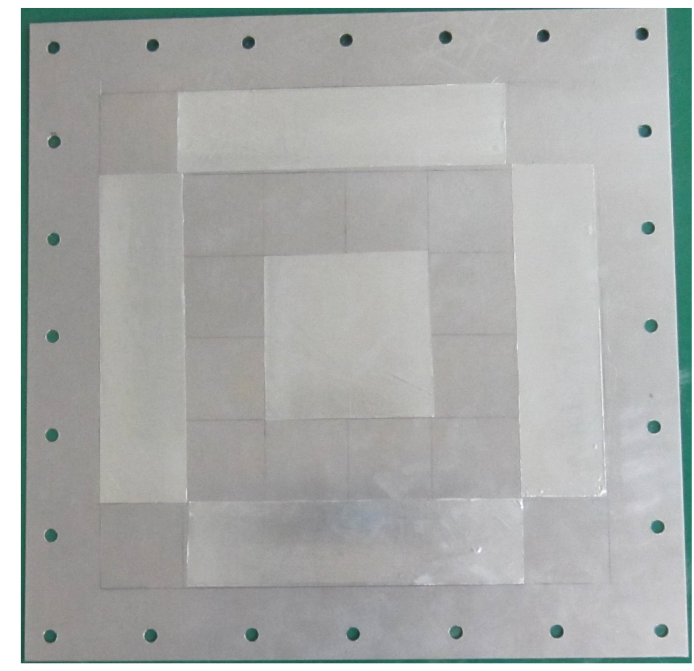

Figure 14. The three flexible plates: (a) aluminum plate, (b) fully CLD treatment plate, and (c) optimized CLD treatment plate. 


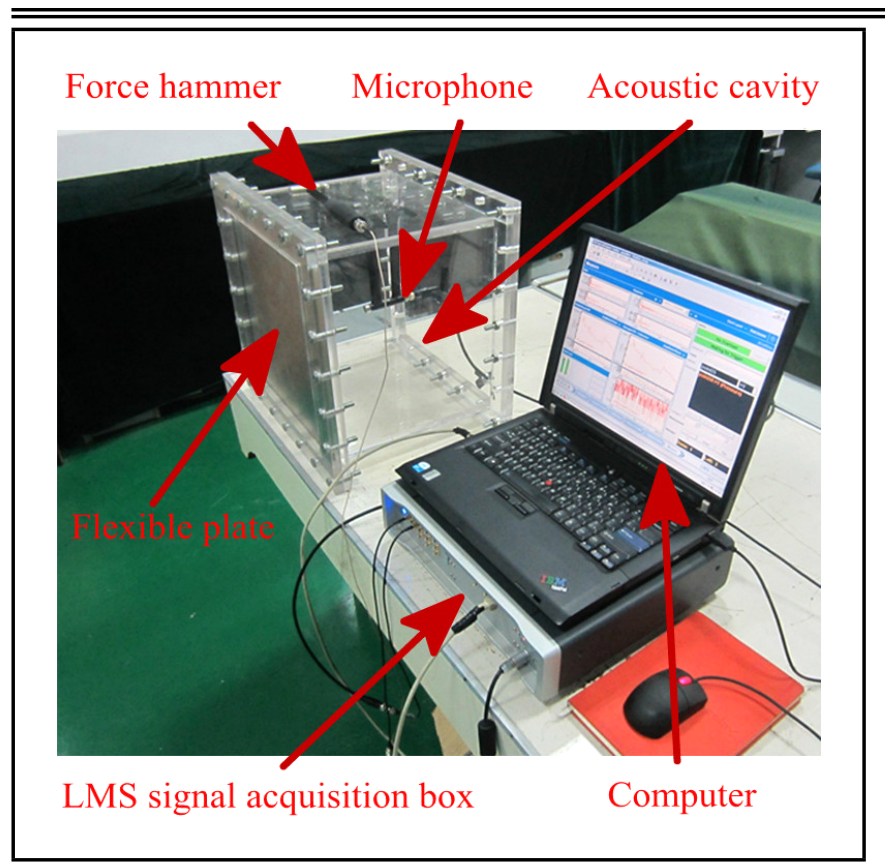

Figure 15. Experimental setup.

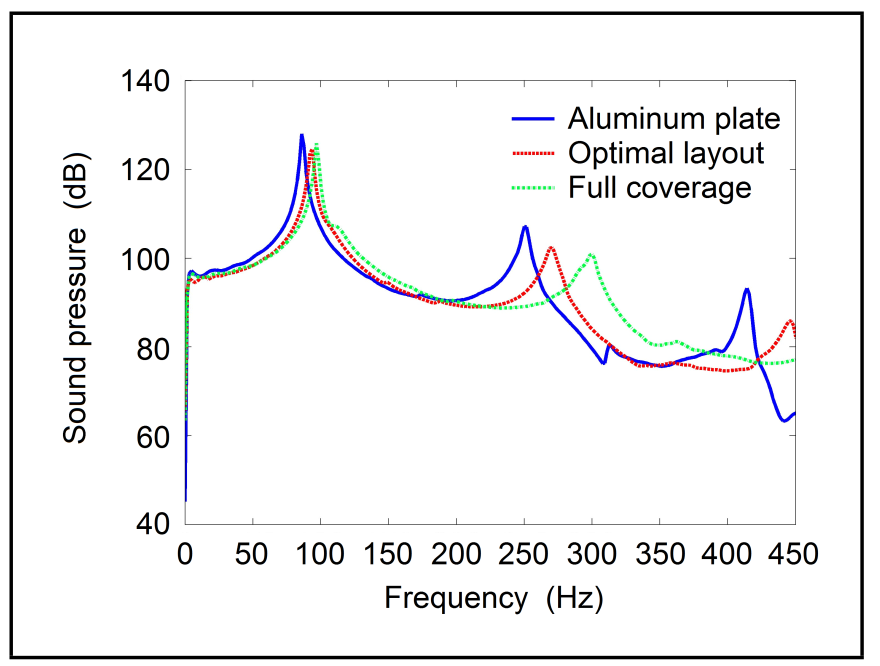

Figure 16. Experimental sound pressure.

\section{REFERENCES}

1 Kerwin, E. M. Damping of flexural waves by a constrained viscoelastic layer, Journal of Acoustical Society of America, 31 (37), 952-962, (1959).

2 Zheng, H., Cai, C., Pau, G. S. H., and Liu, G. R. Minimizing vibration response of cylindrical shells through layout optimization of passive constrained layer damping treatments, Journal of Sound and Vibration, 279 (3-5), 739756, (2005). http://dx.doi.org/10.1016/j.jsv.2003.11.020

3 Alvelid, M. Optimal position and shape of applied damping material, Journal of Sound and Vibration, 310 (4-5), 947965, (2008). http://dx.doi.org/10.1016/j.jsv.2007.08.024

4 Li, Y. N., Xie, R. L., Wang, Y., and Zheng, L. Topology optimization for constrained layer damping material in structures using ESO method, Journal of Chongqing University, 33 (8), 1-6, (2010).
5 Zheng, L., Xie, R. L., Wang, Y., and Li, Y. N. Optimal placement of constrained damping material in structures based on optimality criteria, Journal of Vibration and Shock, 29 (11), 156-159+179, (2010). http://dx.doi.org/10.13465/j.cnki.jvs.2010.11.011

6 Zheng, L., Xie, R. L., Han, Z. M., Adel, E. S., and Baz, A. Topology optimization of damping materials layout to enhance energy dissipation of cylindrical shell, $17^{\text {th }}$ International Congress on Sound and Vibration, (2010).

7 Ansari, M., Khajepour, A., and Esmailzadeh, E. Application of level set method to optimal vibration control of plate structures, Journal of Sound and Vibration, 332 (4), 687700, (2013). http://dx.doi.org/10.1016/j.jsv.2012.09.006

8 Kim, S. Y., Mechefske, C. K., and Kim, I. Y. Optimal damping layout in a shell structure using topology optimization, Journal of Sound and Vibration, 332 (12), 2873-2883, (2013). http://dx.doi.org/10.1016/j.jsv.2013.01.029

9 Mohammadi, F., Sedaghati, R. Vibration analysis and design optimization of viscoelastic sandwich cylindrical shell, Journal of Sound and Vibration, 331 (12), 2729-2752, (2012). http://dx.doi.org/10.1016/j.jsv.2012.02.004

10 Arnold, L. Topology optimization of constrained damping layer treatments, Proceedings of ASME International Mechanical Engineering Congress and Exposition, (2002).

11 Trindade, M. A. Optimization of active-passive damping treatments using piezoelectric and viscoelastic materials, Smart Materials and Structures, 16 (6), 2159-2168, (2007). http://dx.doi.org/10.1088/0964-1726/16/6/018

12 Li, H., Chen, G., and Zhang, B. Topology optimization design of constrained layer damping plate-cavity system considering vibro-acoustic coupling, Journal of Vibration, Measurement and Diagnosis, 31 (5), 586590. (2011). http://dx.doi.org/10.16450/j.cnki.issn.10046801.2011 .05 .003

13 Akl, W., El-Sabbagh, A., Al-Mitani, K., and Baz, A. Topology optimization of a plate coupled with acoustic cavity, International Journal of Solids and Structures, 46 (10), 2060-2074, (2009). http://dx.doi.org/10.1016/j.ijsolstr.2008.05.034

14 Liu, B. S., Zhao, G. Z., and Gu, Y. X. Optimization of acoustic radiation caused by structural vibration of composite laminated plates, Journal of Vibration and Shock, 27 (12), 31-35, (2008). http://dx.doi.org/10.1016/j.ijsolstr.2008.05.034

15 Ciskowski, R. D., Brebbia, C. A. Boundary Element Methods in Acoustics, Computational Mechanics Publications, Southampton, (1991). 